\title{
THE EMERGENCE OF KNOWLEDGE EXCHANGE: AN AGENT-BASED MODEL OF A SOFTWARE MARKET.
}

\author{
EXTENDED ABSTRACT
}

\author{
MARIA CHLI AND PHILIPPE DE WILDE

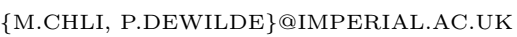 \\ INTELLIGENT SYSTEMS AND NETWORKS GROUP, IMPERIAL COLLEGE LONDON
}

\begin{abstract}
We investigate knowledge exchange among commercial organisations, the rationale behind it and its effects on the market. Knowledge exchange is known to be beneficial for industry, but in order to explain it, authors have used high level concepts like network effects, reputation and trust. We attempt to formalise a plausible and elegant explanation of how and why companies adopt information exchange and why it benefits the market as a whole when this happens. This explanation is based on a multi-agent model that simulates a market. Even though the model does not include any high-level concepts, information exchange naturally emerges during simulations as a successful profitable behaviour. The conclusions reached by this agent-based analysis are twofold: (1) A straightforward set of assumptions is enough to give rise to exchange in a software market. (2) Knowledge exchange is shown to increase the efficiency of the market.
\end{abstract}

\section{INTRODUCTION}

The growth of the Internet as a medium of knowledge exchange has stimulated a lot of scientific interest originating from various disciplines. The willingness of individuals, organisations as well as firms to share information via the Internet has been remarkable. In some sectors like scientific research, the communication of newly acquired knowledge in a field is considered vital for their advancement. On the other hand, in other sectors, the benefits of such exchanges may not be obvious. For instance, it might be considered damaging for pharmaceutical firms to make public any innovations generated by their research and development $(R \& D)$ process. In spite of this view, exchange of intellectual property in some industries occurs frequently and in various different ways. These include the forming of strategic partnerships, the participation in open source software projects and the publication of scientific papers by research labs that are part of commercial firms. In existing literature, [Hagedoorn, 2002], [Lerner and Tirole, 2002] and others justify the decisions of companies to share some of their knowledge using the concepts like trust, network effects and strategic reasons.

We focus on analysing the rationale behind this exchange as well as its effect to the market. This effort fits within the framework of the Digital Business Ecosystem (DBE) project [DBE, 2002]. The DBE project is an attempt to develop a distributed environment which will interlink European Small and Medium Enterprises (SMEs) and foster collaboration between them.

We are interested in analysing the global system properties which emerge from the interactions that occur in a market. We have been using techniques from agent based modelling to simulate the DBE environment. The main aspects of the DBE market are captured in a model where the firms are agents with bounded rationality. This model is then studied using simulations and a number of observations are made. One of the most interesting observations is that exchanges between the agents similar to the ones that happen in real-life arise in the system. This behaviour emerges in the market even though the model does not explicitly account for social issues of trust, network effects or managerial strategies.

\section{An Agent-Based model of the DBE}

Companies are modelled as agents in a multi-agent system. The services the companies provide are modelled as bit strings, with each bit symbolising a feature of the service. Finally, the underlying market is modelled by a set of requests (market needs). A request is a bit string of the same size as a service bit string.

Each company has a population (or portfolio) of services. This population is not static throughout the lifetime of the company. If a service is successful, the company tends to add similar services to the portfolio while unsuccessful services are discarded. The whole process is modelled quite elegantly by a Genetic Algorithm within the portfolio which involves mutation and crossover with survival of the fittest. Through this population each company can choose which market segment(s) it will try to satisfy. The Genetic Algorithm (GA) represents the R\&D businesses perform in order to improve their services.

The fitness of a company equals the fitness of the service it offers. The fitness of a service measures how profitable it is to its owner. The profitability of a service depends on: (1)how close the service is to the market needs (service-request similarity) and (2)how many other services satisfy those needs (limited demand).

In our model, the exchange involves selecting a set of services from one company's portfolio and swapping them with the corresponding set of services of the other company's portfolio. At each time tick, the companies are asked to make 
two decisions. Firstly, they need to decide whether they want to exchange some of their services with one of the other companies. Then, in the case that they have decided to exchange, they need to decide with which fellow company to exchange. A statistical classification algorithm, similar to the one used in [Kirman and Vriend, 2001], is used to model each of the decision problems an individual agent faces.

\section{Summary OF RESUlts}

Simulations of the model outlined above were performed for a very wide range of parameters and initial conditions. The model behaviour, which is quite general, is summarised in this section. The details of the experiments performed as well as graphs and figures have been omitted here for brevity, but will be included in the full paper.

The first observation, which is justified by a series of experiments, is that the practice of information sharing emerges in the market as long as the companies' decisions on whether to exchange is based on their performance in the market. More specifically, it shown that it is more beneficial for the worst performing companies in the market to participate in knowledge exchange activities. In addition to that, our experiments show that it is most beneficial for worst performing companies to engage in exchanges with the best performing ones. While this might an obvious result, it justifies the validity of our model. Another less obvious fact which was observed is that it is very beneficial for the worst performing companies in the market to engage in exchanges among themselves. Such exchanges were shown to have tremendous effects to the performance of the companies involved and even bring about changes in the leadership of the market. Finally, it is shown that when sharing of information is allowed in the market it makes for greater market efficiency levels.

\section{CONCluding REMARKS}

The aim of this work has been to study the rationale as well as the effect of knowledge exchange in economic markets. Sharing of information between commercial firms is considered controversial. Although it is acknowledged that when two companies join forces to develop an innovative product they can both benefit, sharing trade secrets is not undertaken lightly. Our main aim has been to formalise a plausible and elegant explanation of how and why companies adopt information exchange and why it benefits the market as a whole when this happens.

An agent based model of a Digital Business Ecosystem market has been implemented to assist us in understanding the dynamics of the market mechanisms. Firms are modelled as agents with minimal reasoning capabilities. We investigated the properties that emerge from the agent interactions that occur in the market. Specifically, we examined two key characteristics that we observed in the simulations carried out. Namely, the fact that the agents discover themselves that under certain circumstances it is beneficial for them to exchange services and that allowing exchange to take place in the market, makes for greater market efficiency levels.

The technologic infrastructure of the DBE will facilitate the dissemination of knowledge among the member companies, increasing the volume and the speed of the information flowing in the market. As a result, it is expected that it will allow for greater market efficiency levels in comparison to a conventional market. Admittedly, it is difficult to compare the market efficiency of two different markets. However, an interesting result arose when we performed simulations of the DBE contrasting settings in which exchanges among companies were permitted with settings where exchanges were not permitted. Exchanges among companies within the DBE further increase the efficiency of the market, which is in agreement with the common intuition that exchanging information is ultimately beneficial for the entire market.

The second and most important conclusion that emerged from the DBE simulation is that exchanges between the agents similar to the ones that happen in real-life arise naturally in our system. At regular time intervals, the firms were given the chance to decide whether they wanted to choose a partner and swap some of their services. The decision was taken using classifiers, which were separate for each agent. The agents were not pre-programmed or biased in any way to engage in exchanges. The companies, on their own, discovered in which cases exchanging is beneficial for them and what type of partner is the best. Exchange is a practice that emerges, and is not forced upon the agents.

This work does not directly advocate service knowledge as a means of increasing profitability of software companies. Knowledge exchange, is indeed an already existing phenomenon in industry. The results presented merely serve as a demonstration of a parsimonious set of assumptions that give rise to exchange in a software market. In other words, we identify the substance of this phenomenon, ridding it from unnecessary assumptions, like network effects, social issues of trust, or managerial strategies and show the minimal set of assumptions that allow it to emerge.

\section{REFERENCES}

[DBE, 2002] DBE (2002). Annex I - Description of Work, Digital Business Ecosystem. Technical report.

[Hagedoorn, 2002] Hagedoorn, J. (2002). Inter-firm R\&D partnerships: an overview of major trends and patterns since 1960. Research Policy, 31(4):477-492.

[Kirman and Vriend, 2001] Kirman, A. P. and Vriend, N. J. (2001). Evolving market structure: An ACE model of price dispersion and loyalty. Journal of Economic Dynamics and Control, 25(3):459-502.

[Lerner and Tirole, 2002] Lerner, J. and Tirole, J. (2002). Some simple economics of open source. Journal of Industrial Economics, 50:197-234. 